Dermatology 2019;235:255-259

DOI: $10.1159 / 000496968$

\section{Characterization of the Blood Microbiota in Korean Females with Rosacea}

Yeojun Yun ${ }^{a}$ Han-Na Kim $^{\text {a }}$ Yoosoo Chang ${ }^{\text {b-d }}$ Yunho Lee Seungho Ryu ${ }^{\text {b-d }}$ Hocheol Shin ${ }^{b, f}$ Won-Serk Kim ${ }^{\text {e Hyung- }}$ Lae Kim $^{\text {a }}$ Jae-Hui Nam ${ }^{\text {e, g }}$

a Department of Biochemistry, Ewha Womans University School of Medicine, Seoul, South Korea; ${ }^{b}$ Center for Cohort Studies, Total Healthcare Center, Kangbuk Samsung Hospital, Sungkyunkwan University School of Medicine, Seoul, South Korea; ${ }^{\mathrm{C}}$ Department of Occupational and Environmental Medicine, Kangbuk Samsung Hospital, Sungkyunkwan University School of Medicine, Seoul, South Korea; ${ }^{\mathrm{d}}$ Department of Clinical Research Design \& Evaluation, SAIHST, Sungkyunkwan

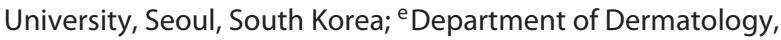
Kangbuk Samsung Hospital, Sungkyunkwan University School of Medicine, Seoul, South Korea; ${ }^{f}$ Department of Family Medicine, Kangbuk Samsung Hospital, Sungkyunkwan University School of Medicine, Seoul, South Korea; ${ }^{9}$ Veterans Medical Research Institute, Veterans Health Service Medical Center, Seoul, South Korea

\section{Keywords}

Bacteria $\cdot$ Blood $\cdot$ Microbiota $\cdot$ Metagenome $\cdot$ Rosacea

Rosacea is a chronic, intractable skin disease that is generally accompanied by vascular dilation and subsequent inflammation of the face [1]. Rosacea has been reported to occur among people of diverse races throughout the world, and its prevalence varies widely from less than $1 \%$ to $20 \%$ according to the study country. The gender distribution is slightly female-predominant [2]. The symptoms of rosacea comprise a complex combination of erythema, papulopustules, telangiectases, or ocular redness. Rosacea may be a proxy for systemic disease, rather than a mere skin disorder, as it has been linked with metabolic, cardiovascular, autoimmune, and chronic kidney diseases $[3,4]$. However, most of the relevant data are from epidemiologic studies without basic experimental details. Thus, further evidence must be sought to support the hypothesis that rosacea is associated with systemic inflammation and systemic diseases.

The analysis of $16 \mathrm{~S}$ ribosomal RNA (rRNA) fragments from the human microbiome is emerging as a novel tool for understanding the pathogenesis of human disease. Metagenomic approaches for investigating microbial genomes are being used to determine the potential roles of the microbiotas of the gut, skin, blood, and other human derivatives in chronic inflammatory diseases [5-7]. Of note, a new insight was made into the pathologic significance of the blood microbiota that had been overlooked in conventional culture-based research. By identifying identical bacterial gene sequences at two different sites, Huang et al. [8] determined that Helicobacter pylori exists in the peripheral blood as well as in the gastric mucosa of patients with duodenal ulcers.

Rosacea is associated not only with various gastrointestinal diseases, but also with gut dysbiosis [9-11]. Indeed, our previous cross-sectional study demonstrated that the gut microbiota was altered in rosacea subjects [11]. Given the aberrant composition of the gut microbiota in rosacea subjects and the putative role of the blood microbiota in comorbid diseases, there may be a connection between the gut, blood, and skin inflammation.

The objective of this study was to detect differences in the blood microbiota between Korean female rosacea subjects and rosaceafree controls.

In 2014, this study was performed to assess the differences in the blood microbiota between rosacea and rosacea-free subjects. We selected age- and body mass index-matched groups of rosacea patients and control subjects from a total of 282 female subjects. Rosacea patients were identified based on a history of rosacea or a definite manifestation of rosacea. Individuals with a history of antibiotic use within 6 weeks or probiotic use within 4 weeks, a history of diabetes mellitus, or a low glomerular filtration rate $(<60$ $\mathrm{mL} / \mathrm{min}$ ) were excluded $(n=23)$. Ultimately, 10 subjects with rosacea and 30 controls were included.

The blood samples were collected by peripheral vein puncture in sterile conditions after cleansing skin with an alcohol swab. One purple-top (EDTA) vacutainer containing whole blood was collected from each subject and immediately stored at $-4{ }^{\circ} \mathrm{C}$. On blood collecting, genomic DNA was extracted from whole blood samples with the use of a commercial isolation kit (G-DEX IIb Genomic DNA Extraction Kit for Blood; iNtRON Biotechnology, Korea) according to the manufacturer's protocols. The buffy coat consisting mainly of leucocytes was used for DNA extraction from blood samples. Variable V3 and V4 regions of the 16S rRNA gene were amplified with the universal primers 341F (5' TCG TCG GCA GCG TCA GAT GTG TAT AAG AGA CAG CCT ACG GGN GGC WGC AG $3^{\prime}$ ) and 805R (5' GTC TCG TGG GCT CGG AGA TGT GTA TAA GAG ACA G GA CTA CHV GGG TAT CTA ATC C $3^{\prime}$ ), with each primer modified to contain a unique 8-nt barcode index by combination with the Nextera XT DNA Library Preparation kit (Illumina, San Diego, CA, USA). The PCR reactions contained $5 \mathrm{ng} / \mu \mathrm{L}$ DNA template, $2 \times \mathrm{KAPA}$ HiFi HotStart Ready Mix (KAPA Biosystems, Wilmington, MA, USA), and 2 pmol of each primer. The reaction conditions consisted of an initial incubation at $95^{\circ} \mathrm{C}$ for $3 \mathrm{~min} ; 25$ cycles of $95^{\circ} \mathrm{C}$ for $30 \mathrm{~s}$, $55^{\circ} \mathrm{C}$ for $30 \mathrm{~s}$, and $72^{\circ} \mathrm{C}$ for $30 \mathrm{~s}$; and a final extension incubation at $72^{\circ} \mathrm{C}$ for $5 \mathrm{~min}$. After PCR clean-up and index PCR, sequencing was performed on the Illumina MiSeq platform according to the manufacturer's specifications $[12,13]$.

\section{KARGER}

(c) 2019 S. Karger AG, Basel

E-Mail karger@karger.com

www.karger.com/drm
Jae-Hui Nam, MD

Department of Dermatology, Kangbuk Samsung Hospital Sungkyunkwan University School of Medicine

29 Saemunan-ro, Jongno-gu, Seoul 03181 (South Korea)

E-Mail guitactor@gmail.com 


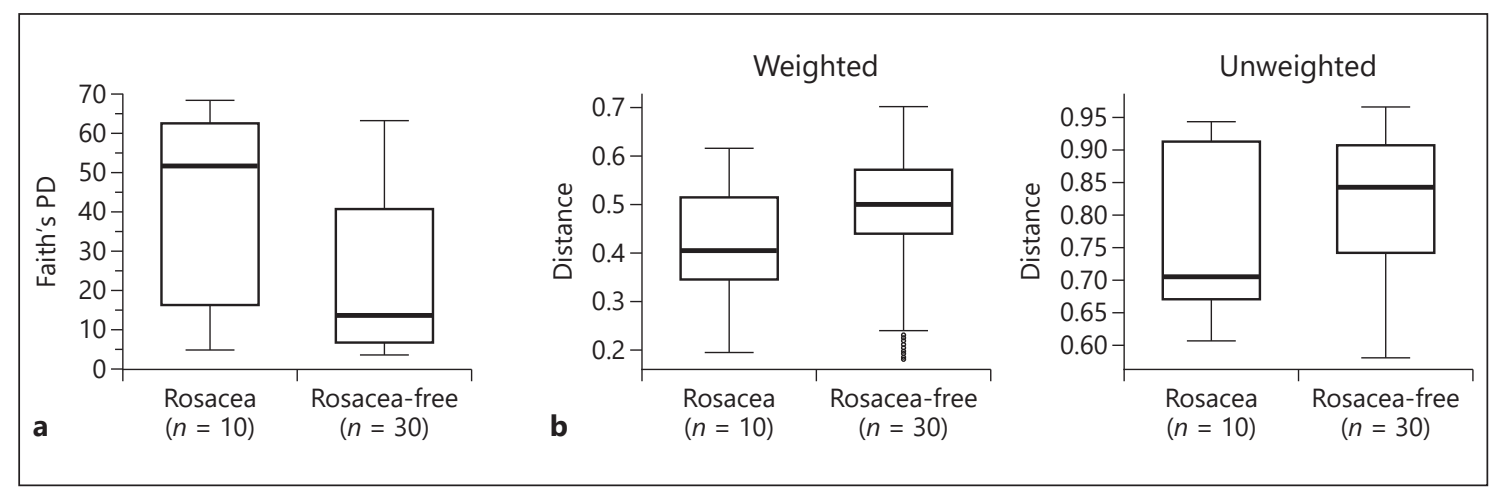

Fig. 1. Diversity assessment of the blood microbiota in rosacea. a The alpha diversity between rosacea patients and rosacea-free controls, measured as Faith's phylogenetic diversity (Faith's PD), was of marginal significance $(p=0.09)$. $\mathbf{b}$ The beta diversity between rosacea patients and controls was calculated with weighted (left) $(p=$ $0.01)$ and unweighted (right) $(p=0.07)$ UniFrac metrics. Accordingly, rosacea patients' microbiotas were more similar to those of other rosacea patients than to those of controls.

The DADA2 pipeline [14] within the QIIME2 package (https:// qiime2.org) [15] was used to filter low-quality and chimera errors and generate unique sequence variants. Because the "operational taxonomic units (OTUs)" resulting from DADA2 are created through the grouping of unique sequences, these are the equivalent of $100 \%$ OTUs, and are generally referred to as sequence variants. QIIME2 was used to construct the FeatureTable, which is the equivalent of the BIOM table and the representative sequence file. The sequencing depth ranged from 6,554 to 136,763 reads per sample (total frequency $=1,415,559$ ) and 9,705 features. For diversity analysis, we rarefied the data to 5,000 sequences per sample. The sequences were mapped at $99 \%$ sequence identity to an optimized version of the GreenGenes database (version 13.8) containing the $\mathrm{V} 3-\mathrm{V} 4$ region to determine taxonomies.

Exploratory and differential microbial composition analyses were conducted in QIIME2, version 2017.10. We used Shannon's diversity index, which aims to measure diversity by accounting for evenness and richness [16]: evenness, which measures community evenness, and the "observed OTUs," which reflect the actual number of different taxa observed in a sample. Additionally, alpha diversity was measured with a phylogenetic diversity measurement, Faith's phylogenetic diversity, often referred to as PD [17]. The Kruskal-Wallis test was used to estimate the differences between the two groups. Unweighted and weighted UniFrac distances [18] were used as a phylogenetic method to determine the dissimilarity between the communities in terms of their membership and structure. A permutational ANOVA (PERMANOVA) pairwise test for the distance matrix was performed via 999 Monte Carlo permutations and false discovery rate multiple correction. Analysis of composition of microbiomes (ANCOM) in QIIME2 was applied to evaluate the differential abundance of bacterial taxa between the sample groups [19].

The rosacea cases and controls were matched for age and BMI and were all females. The erythematotelangiectatic subtype was the most frequent, followed by papulopustular rosacea. In alpha diversity analysis, there was a marginally significant difference $(p=0.09)$ between the rosacea and control groups when Faith's phylogenet- ic diversity was used, whereas other alpha diversity indexes (observed OTUs, Shannon's diversity index, and evenness) did not differ between the two groups (Fig. 1a; online suppl. Fig. S1; see www.karger.com/doi/10.1159/000496968 for all online suppl. material). In terms of beta diversity distances, the overall blood microbial communities of rosacea patients were distinguishable from those of rosacea-free controls, according to weighted $(p=0.01)$ and unweighted ( $p=0.07$ ) UniFrac metrics (Fig. 1b). Additionally, on the principal coordinates plot, the blood microbiota from the two groups clustered separately to some extent, especially when delineated by weighted UniFrac metrics (online suppl. Fig. S1).

To analyze the differential abundance of blood microbiota between rosacea patients and controls, we conducted ANCOM. Two bacterial taxa at the family level and 14 bacterial taxa at the genus level differed in abundance between rosacea patients and controls. For the identification of the differential abundance of blood microbiota, the $\mathrm{W}$ value is used to evaluate the null hypothesis of two groups having no significant difference in ANCOM. In detail, the higher the $\mathrm{W}$ value of a species, the more significantly the species differs across groups [19]. At the family level, it was found that Chromatiaceae (Gammaproteobacteria class, Alteromonadales order, Chromatiaceae family) and Fusobacteriaceae (Fusobacteriia class, Fusobacteriales order, Fusobacteriaceae family) were significantly elevated in rosacea patients (W value $>100$ ) (Fig. 2; online suppl. Fig. S2). Regarding blood microbes with high W values $(>100)$ at the genus level, Rheinheimera (Gammaproteobacteria class, Alteromonadales order, Chromatiaceae family) was the most significantly enriched in rosacea subjects. The other blood microbiota identified at the genus level were Sphingobium, Tissierellaceae family unknown genus, Paracoccus, Rhodovulum, Marinobacter, Chthoniobacteraceae family unknown genus, Methanobacterium, Armatimonadaceae family unknown genus, Clostridiaceae family unknown genus, Fusobacterium, and Citrobacter ( $\mathrm{W}$ value $<30$ ) (online suppl. Fig. S2). The significant taxa that rejected the null hypothesis in terms of their differential abundance between the rosacea and control groups in ANCOM are shown in Table 1. 
Fig. 2. Relative abundance of blood bacterial taxa at the family level in subjects with or without rosacea. The Chromatiaceae family (a) and Fusobacteriaceae family (b) were enriched in blood samples from rosacea patients.

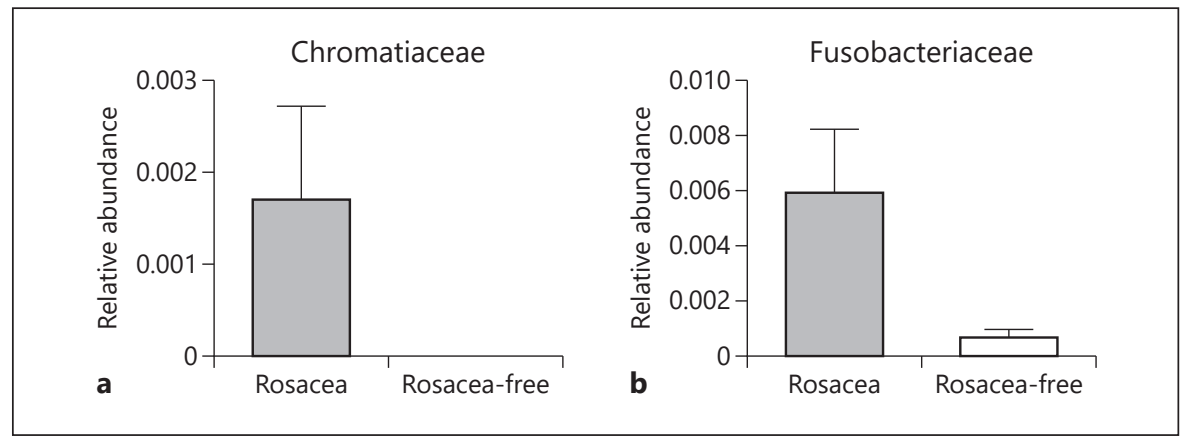

Table 1. Abundance of blood bacterial taxa at the family level and the genus level in rosacea patients versus controls based on the analysis of composition of microbiomes (ANCOM)

a ANCOM results at the family level

\begin{tabular}{|c|c|c|c|c|c|c|}
\hline Kingdom & Phylum & Class & Order & Family & & W value \\
\hline Bacteria & Proteobacteria & Gammaproteobacteria & Alteromonadales & Chromatiaceae & & 126 \\
\hline Bacteria & Fusobacteria & Fusobacteriia & Fusobacteriales & Fusobacteriaceae & & 109 \\
\hline \multicolumn{7}{|c|}{ b ANCOM results at the genus level } \\
\hline Kingdom & Phylum & Class & Order & Family & Genus & $\mathrm{W}$ value \\
\hline Bacteria & Proteobacteria & Gammaproteobacteria & Alteromonadales & Chromatiaceae & Rheinheimera & 203 \\
\hline Bacteria & Proteobacteria & Alphaproteobacteria & Sphingomonadales & Sphingomonadaceae & Sphingobium & 26 \\
\hline Bacteria & Firmicutes & Clostridia & Clostridiales & Tissierellaceae & & 24 \\
\hline Bacteria & Proteobacteria & Alphaproteobacteria & Rhodobacterales & Rhodobacteraceae & Paracoccus & 19 \\
\hline Bacteria & Proteobacteria & Gammaproteobacteria & Alteromonadales & Alteromonadaceae & Marinobacter & 11 \\
\hline Bacteria & Verrucomicrobia & Spartobacteria & Chthoniobacterales & Chthoniobacteraceae & & 11 \\
\hline Bacteria & Proteobacteria & Alphaproteobacteria & Rhodobacterales & Rhodobacteraceae & Rhodovulum & 9 \\
\hline Archaea & Euryarchaeota & Methanobacteria & Methanobacteriales & Methanobacteriaceae & Methanobacterium & 7 \\
\hline Bacteria & Armatimonadetes & Armatimonadia & Armatimonadales & Armatimonadaceae & & 5 \\
\hline Bacteria & Firmicutes & Clostridia & Clostridiales & Clostridiaceae & & 5 \\
\hline Bacteria & Fusobacteria & Fusobacteriia & Fusobacteriales & Fusobacteriaceae & Fusobacterium & 5 \\
\hline Bacteria & Proteobacteria & Gammaproteobacteria & Enterobacteriales & Enterobacteriaceae & Citrobacter & 5 \\
\hline
\end{tabular}

We found that rosacea was related to an aberrant blood microbiota composition. Also, the blood microbiota of rosacea patients was distinct from and more diverse than that of controls. Fusobacteriaceae and Chromatiaceae were prevalent at the family level, and at the genus level, Rheinheimera was the most significantly abundant in rosacea patients' blood. To the best of our knowledge, ours is the first study using a metagenomics approach to detect alterations in the blood microbiota of rosacea patients.

There has been increasing interest in Fusobacterium residing in the colon because Fusobacterium acts as an immunologic trigger in inflammatory diseases. It is known that Fusobacterium, which is Gram-negative bacteria, is indigenous to the oral cavity and can be readily found in the colon under inflamed conditions [20]. In a study where Fusobacterium and relevant inflammatory markers were investigated in subjects with colorectal adenoma, interleukin (IL)-10 and TNF- $\alpha$ were elevated in the colonic mucosa in proportion to the abundance of Fusobacterium in the colon, and IL- 6 and IL-17 levels exhibited a statistically insignificant positive correlation with the abundance of Fusobacterium. Growing inflammation associated with Fusobacterium colonization seems to lead to the development of colorectal adenoma. As such, Fusobacterium may contribute to colorectal carcinogenesis [21]; indeed, Fusobacteri$u m$ was enriched in colorectal cancer tissue compared with normal colonic tissue [22]. Whether Fusobacterium in the bowel can translocate into the bloodstream is uncertain so far. However, given that Fusobacterium varium, which inhabits the enteral mucosa, was highly detected in colon tissue samples from patients with active ulcerative colitis (UC), along with increasing serum antibody lev- 
els of F. varium, a link between UC and Fusobacterium remains plausible [23]. The association between rosacea and UC has been well established by previous studies, based on the increased prevalence of UC in rosacea patients and the shared genetic background between rosacea and inflammatory bowel disease [10,24]. In this regard, Fusobacterium may account for blood dysbiosis as well as enteral inflammation accompanied with rosacea, and thus may be a link between rosacea and UC.

Chromatiaceae, which is Gram-negative purple sulfur bacteria, inhabits aquatic anaerobic environments and requires sulfur compounds for anoxygenic photosynthesis [25, 26]. Rheinheimera, which belongs to Chromatiaceae, is a genus of oxidase- and catalase-positive, aerobic bacteria that is distributed in water, soil, and plants [27]. The pathophysiological roles of these bacteria in humans have not yet been elucidated. Nevertheless, it is presumable that alterations in the composition of the candidate blood microbiota stimulate inflammatory mechanisms, since the outer membrane of Gram-negative bacteria is composed of lipopolysaccharide. Lipopolysaccharide elicits innate immune responses via Tolllike receptor 4 and is associated with various chronic inflammatory diseases, including atherosclerosis, arthritis, and cancer [28].

We previously observed that changes in the gut microbiota were associated with rosacea. In the intestine, Peptococcaceae family unknown genus and Methanobrevibacter were reduced and Acidaminococcus and Megasphaera were elevated in rosacea subjects compared to rosacea-free individuals, even after adjustment for covariates [11]. Contrary to what was anticipated, the gut bacteria linked to rosacea differed from the blood bacteria that were altered in rosacea subjects in this study. As indicated in a previous study investigating blood and gut dysbiosis in patients with liver failure, bacterial profiles can differ between the blood and the gut despite the correlation between the two [7]. Moreover, differences in the study design and rosacea subjects may have influenced the study results.

For now, only a few investigations into blood microbial genomes in dermatologic diseases have been conducted. In a casecontrol study of hidradenitis suppurativa, there was no different bacterial composition in peripheral blood between affected and healthy individuals. Therefore, it was concluded that bacteremia does not seem to contribute to systemic symptoms in hidradenitis suppurativa patients [29]. However, the study has several limitations, including the small sample size, and thus warrants further study.

Our study had several limitations: the small sample size and the uncertain cause-effect relationship between certain bacteria and rosacea.

To conclude, our study suggests that changes in the blood microbiota are associated with rosacea. Although these results warrant validation in experiment-based and/or larger longitudinal studies, our use of a novel technology has broadened our perspective on the pathogenesis of rosacea and may contribute to future diagnostic and therapeutic efforts.

Key Message

Changes in the blood microbiota are associated with rosacea in Korean females.

\section{Acknowledgments}

We acknowledge the computing resources of the Global Science Experimental Data Hub Center (GSDC) Project and the Ko- rea Research Environment Open NETwork (KREONET) in the Korea Institute of Science and Technology Information (KISTI).

The data sets used and/or analyzed during the current study are available from the Clinical and Omics data archives (CODA) in the Korea National Institute of Health (KNIH). The raw 16S rRNA gene sequencing data sets (fastq files), and age and gender information per sample are available in the public repository, CODA (Clinical and Omics data archives, http://coda.nih.go.kr/coda/frt/ index.do, accession number: R000635) at the KNIH.

\section{Statement of Ethics}

This study protocol was approved by the Institutional Review Board at Kangbuk Samsung Hospital (IRB number: 2013-01-24512). All procedures performed in studies involving human participants were in accordance with the ethical standards of the institutional and/or national research committee and with the 1964 Helsinki declaration and its later amendments or comparable ethical standards. Informed consent was obtained from all individual participants included in the study.

\section{Disclosure Statement}

The authors have no conflict of interest to declare.

\section{Funding Sources}

This study was supported by National Research Foundation of Korea (NRF) grants funded by the Korean government (NRF2017R1D1A1B03032776 and NRF-2016R1A6A3A11932719).

\section{Author Contributions}

Yeojun Yun, Han-Na Kim, and Jae-Hui Nam analyzed the data and wrote the manuscript. Jae-Hui Nam proposed the study and approved the final paper. Yunho Lee and Won-Serk Kim assisted with data acquisition and reviewed the article. Hocheol Shin, Yoosoo Chang, and Seungho Ryu designed the original form of the study and collected the data. Hyung-Lae Kim aided in the interpretation of the data.

\section{References}

1 Spoendlin J, Voegel JJ, Jick SS, Meier CR. Risk of rosacea in patients with diabetes using insulin or oral antidiabetic drugs. J Invest Dermatol. 2013 Dec;133(12):2790-3.

2 Tan J, Berg M. Rosacea: current state of epidemiology. J Am Acad Dermatol. 2013 Dec;69(6 Suppl 1):S27-35.

3 Chiu HY, Huang WY, Ho CH, Wang JJ, Lin SJ, Hsu YW, et al. Increased risk of chronic kidney disease in patients with rosacea: A nationwide population-based matched cohort study. PLoS One. 2017 Oct; 12(10):e0180446.

4 Egeberg A, Hansen PR, Gislason GH, Thyssen JP. Clustering of autoimmune diseases in patients with rosacea. J Am Acad Dermatol. 2016 Apr; 74(4):667-72.e1.

5 Grice EA. The skin microbiome: potential for novel diagnostic and therapeutic approaches to cutaneous disease. Semin Cutan Med Surg. 2014 Jun;33(2):98-103.

6 Hill-Burns EM, Debelius JW, Morton JT, Wissemann WT, Lewis MR, Wallen ZD, et al. Parkinson's disease and Parkinson's disease medications have distinct signatures of the gut microbiome. Mov Disord. 2017 May;32(5):739-49.

7 Lelouvier B, Servant F, Païssé S, Brunet AC, Benyahya S, Serino M, et al. Changes in blood microbiota profiles associated with liver fibrosis in obese patients: A pilot analysis. Hepatology. 2016 Dec;64(6):2015-27. 
8 Huang Y, Fan XG, Tang ZS, Liu L, Tian XF, Li N. Detection of Helicobacter pylori DNA in peripheral blood from patients with peptic ulcer or gastritis. APMIS. 2006 Dec;114(12):851-6.

9 Parodi A, Paolino S, Greco A, Drago F, Mansi C, Rebora A, et al. Small intestinal bacterial overgrowth in rosacea: clinical effectiveness of its eradication. Clin Gastroenterol Hepatol. 2008 Jul;6(7):759-64.

10 Egeberg A, Weinstock LB, Thyssen EP, Gislason GH, Thyssen JP. Rosacea and gastrointestinal disorders: a population-based cohort study. Br J Dermatol. 2017 Jan;176(1):100-6.

11 Nam JH, Yun Y, Kim HS, Kim HN, Jung HJ, Chang Y, et al. Rosacea and its association with enteral microbiota in Korean females. Exp Dermatol. 2018 Jan;27(1):37-42.

12 Fadrosh DW, Ma B, Gajer P, Sengamalay N, Ott S, Brotman RM, et al. An improved dual-indexing approach for multiplexed 16S rRNA gene sequencing on the Illumina MiSeq platform. Microbiome. 2014 Feb;2(1): 6.

13 Kozich JJ, Westcott SL, Baxter NT, Highlander SK, Schloss PD. Development of a dual-index sequencing strategy and curation pipeline for analyzing amplicon sequence data on the MiSeq Illumina sequencing platform. Appl Environ Microbiol. 2013 Sep;79(17):5112-20.

14 Callahan BJ, McMurdie PJ, Rosen MJ, Han AW, Johnson AJ, Holmes SP. DADA2: high-resolution sample inference from Illumina amplicon data. Nat Methods. 2016 Jul;13(7):581-3.

15 Caporaso JG, Kuczynski J, Stombaugh J, Bittinger K, Bushman FD, Costello EK, et al. QIIME allows analysis of high-throughput community sequencing data. Nat Methods. 2010 May;7(5):335-6.

16 Jost L. Partitioning diversity into independent alpha and beta components. Ecology. 2007 Oct;88(10):2427-39.

17 Faith DP, Baker AM. Phylogenetic diversity (PD) and biodiversity conservation: some bioinformatics challenges. Evol Bioinform Online. 2007 Feb;2:121-8.

18 Lozupone C, Lladser ME, Knights D, Stombaugh J, Knight R. UniFrac: an effective distance metric for microbial community comparison. ISME J. 2011 Feb;5(2):169-72.
19 Mandal S, Van Treuren W, White RA, Eggesbø M, Knight R, Peddada SD. Analysis of composition of microbiomes: a novel method for studying microbial composition. Microb Ecol Health Dis. 2015 May;26:27663.

20 Swidsinski A, Dörffel Y, Loening-Baucke V, Theissig F, Rückert JC, Ismail $\mathrm{M}$, et al. Acute appendicitis is characterised by local invasion with Fusobacterium nucleatum/necrophorum. Gut. 2011 Jan;60(1):34-40.

21 McCoy AN, Araújo-Pérez F, Azcárate-Peril A, Yeh JJ, Sandler RS, Keku TO. Fusobacterium is associated with colorectal adenomas. PLoS One. 2013;8(1):e53653.

22 Kostic AD, Gevers D, Pedamallu CS, Michaud M, Duke F, Earl AM, et al. Genomic analysis identifies association of Fusobacterium with colorectal carcinoma. Genome Res. 2012 Feb;22(2):292-8.

23 Ohkusa T, Sato N, Ogihara T, Morita K, Ogawa M, Okayasu I. Fusobacterium varium localized in the colonic mucosa of patients with ulcerative colitis stimulates species-specific antibody. J Gastroenterol Hepatol. 2002 Aug;17(8):849-53.

24 Chang AL, Raber I, Xu J, Li R, Spitale R, Chen J, et al. Assessment of the genetic basis of rosacea by genome-wide association study. J Invest Dermatol. 2015 Jun;135(6):1548-55.

25 Frigaard NU, Dahl C. Sulfur metabolism in phototrophic sulfur bacteria. Adv Microb Physiol. 2009;54:103-200.

26 Pfennig N, Trüper HG. The family chromatiaceae. In: Balows A, Trüper HG, Dworkin M, Harder W, Schleifer K, editors. The prokaryotes. New York (NY): Springer; 1992. pp. 3200-21.

27 Chen WM, Lin CY, Young CC, Sheu SY. Rheinheimera aquatica sp. nov., an antimicrobial activity producing bacterium isolated from freshwater culture pond. J Microbiol Biotechnol. 2010 Oct;20(10):1386-92.

28 Chilton PM, Embry CA, Mitchell TC. Effects of Differences in Lipid A Structure on TLR4 Pro-Inflammatory Signaling and Inflammasome Activation. Front Immunol. 2012 Jun;3:154.

29 Ring HC, Thorsen J, Saunte DM, Lilje B, Bay L, Theut Riis P, et al. Moderate to severe hidradenitis suppurativa patients do not have an altered bacterial composition in peripheral blood compared to healthy controls. J Eur Acad Dermatol Venereol. 2018 Jan;32(1):125-8. 